\title{
Supernova Spectra
}

\author{
Massimo Turatto \\ Osservatorio Astronomico di Padova, INAF, vicolo dell'Osservatorio 5, 35122 \\ Padova, Italy; \\ turatto@pd.astro.it
}

\begin{abstract}
Summary. In this paper are summarized the main advances of the last years in the field of SN spectra. The arguments against a monodimensional sequence for SNIa are discussed as well as the efforts to improve the temporal and spectral coverage of this kind of SNe, with the aim to understand the physics of the explosions for their use as cosmological distance indicators. Although variety is the main character of core-collapse SNe, we have been recently surprised by both exceptionally underand over-energetic explosions. The main properties of these two extreme subclasses are here reviewed.
\end{abstract}

\section{Introduction}

Spectra provide most of the physical information on supernovae. Their early analysis has shown a variety of forms and evolutions. Indeed the identification of different spectral lines reveals the presence of several different ions in the layers above the photosphere, suggesting the existence of various progenitors and explosion models. Spectra also allow us the direct determination of the physical conditions of the emitting regions, while the line profiles provide the kinematics.

Supernova spectra evolve rapidly: the effective temperature and expansion velocity decrease, and the spectral lines change. Indeed, because of the expansion, the photosphere recedes into the ejecta and different layers are progressively exposed. In this way the analysis of SN spectra taken at different epochs allows us, at least in principle, to make the tomography of the exploding stars and to reconstruct their entire structures. A major limitation is that spectral features in SN spectra are generally blended due to the large expansion velocities and the full information can be extracted only with the use of complex spectral modeling which try to deal in a consistent way the luminosity, abundances, stratification, temperature, velocity and time evolution.

In general, the light from $\mathrm{SNe}$ is traveling for several megaparsecs before reaching the observers thus contains information on the circumstellar, interstellar and intergalactic matter it has passed through. In principle, high resolution spectroscopy might allow us to determine the distribution and physical conditions of the intervening medium and, in turn, the total reddening and extinction suffered by the light. 
Finally, in the recent years spectral observations on faint, distant of SNIa have provided sufficiently accurate redshifts which, once coupled with the photometry, have inspired a new vision of the Universe we are living in.

This review discusses a number of hot topics concerning SN spectra while reference is given to other reviews for the detailed description of the properties and evolution of various spectral types $[13,57,59]$.

\section{A Spectral Sequence for SNIa?}

SNIa explode in all types of galaxies, in ellipticals as well as in spirals, but in the latter are not closely associated with the spiral arms as other SN types. The spectra are characterized by lines of intermediate mass elements such as $\mathrm{Ca}, \mathrm{O}$, $\mathrm{Si}$ and $\mathrm{S}$ during the peak phase, and by the absence of $\mathrm{H}$ at any time. With age the contribution of the $\mathrm{Fe}$ lines increases and several months past maximum the spectra are dominated by $[\mathrm{Fe} \mathrm{II]} \mathrm{and} \mathrm{[Fe} \mathrm{III]}$ lines. The overall homogeneous spectroscopic and photometric behavior has led to a general consensus that they are associated with the thermonuclear explosions of white dwarfs [5].

During the last decade a new scenario for the SNIa has been progressively developed. In particular, a correlation between the peak luminosity and the shape of the early light curve was found, with brighter objects having a rate of decline slower than dimmer ones $[44,45,46,47]$. An analogous spectroscopic sequence has been found [41], according to which the absolute magnitude of SNIa and, in turn, the rate of decline, is correlated to $\mathcal{R}(\mathrm{SiII})$, the ratio of the depths of two absorption features at 5800 and $6100 \AA$, usually attributed to Si II. Synthetic spectra modeling indicates that most of the spectral differences are caused by variations in the effective temperatures, likely due to different amounts of ${ }^{56} \mathrm{Ni}$ produced in the explosions. The finding that fainter SNe show slow expansion velocities both at early [4] and later epochs [54] is consistent with such scenario.

In first approximation all SNIa can be accommodated into such oneparameter sequence which can be regarded as a sequence of explosion strengths. Within such scheme fit the bright, slowly declining SN 1991T, which did not exhibit Si II or Ca II absorption lines in the premaximum spectra but had a normal behavior starting one month after maximum, and the faint, intrinsically red and fast declining SN $1991 \mathrm{bg}$, which showed a slow expansion velocities and a deep trough around $4200 \AA$ produced by Ti II.

In the last years, however, new findings have challenged such a monoparametric sequence. Hatano et al. [22] have shown that the $\mathcal{R}(\mathrm{SiII})$ does not correlated with the photospheric velocity deduced from the Si II $\lambda 6355 \mathrm{ab}-$ sorption, as one would expect, and propose the existence of two or more explosion mechanisms as possible explanation for the lack of correlation. Also, a recent reanalysis [3] has shown that while the $\mathcal{R}(\mathrm{SiII})$ vs. $\Delta m_{15}$ relation 
holds for $\Delta m_{15} \geq 1.2$, at smaller values it breaks down, thus questioning the correspondence between spectroscopic and photometric parameters.

A number of objects have now good sequences of spectra, some starting very early after the explosion, which make possible to study the temporal evolution of $\mathcal{R}(\mathrm{SiII})$. It is found that before maximum $\mathcal{R}$ (SiII) exhibits a dramatic evolution with opposite trends for various objects [3].

Moreover, objects with similar decline rates can show different spectral features, especially before maximum. In Fig. 2 the spectra of four objects taken about one week before $\mathrm{B}$ maximum are compared. The spectrum of SN 2000E $\left(\Delta m_{15}=0.94 \mathrm{mag}(100 \mathrm{~d})^{-1}\right)$ shows noticeable differences with respect the two $\mathrm{SNe}$ at the bottom, having the same decline rates, but is very similar to that of SN $1990 \mathrm{~N}\left(\Delta m_{15}=1.05\right)$. Not only it differs from that of SN $1991 \mathrm{~T}\left(\Delta m_{15}=0.94\right)$ which is well known for not displaying at this epoch the characteristic Si II line but shows disturbing differences with the normal SN 1999ee $\left(\Delta m_{15}=0.92\right)$. In particular, the profile of the Si II $6355 \lambda$ line is definitely broader in SN 1999ee indicating the presence of $\mathrm{Si}$ II at high velocity. Also the SiII $4130 \lambda$ line, which can be used for classifying high redshift SNe as type Ia, is nearly washed out. Note also that the analysis of the Ca II IR triplet of SN 1999ee before maximum (Mazzali private communication) requires high velocity material in analogy to what found for 2001el [53].

Two other odd SNIa are 2000cx and 2002cx which do not fit into any photometric and spectroscopic sequence still showing the main characteristics of type Ia SNe [30, 31].

\section{Trends in SNIa Spectroscopy}

More and better data are required to clarify which parameters govern the SNIa explosions and to validate the proposed progenitor scenarios. To solve these questions large collaborations have born which have as immediate goal the intensive, multiwavelength monitoring of nearby SNIa.

Examples of such intensive monitoring are the studies of SNe 1999ee [19] and 2002 bo [3], for which the rise to maximum has been sampled daily starting about two weeks before maximum. The analysis of these spectra has allowed the discovery of strong time evolutions of the ratio $\mathcal{R}$ (SiII) with individual behaviors for each SNIa (cf. Fig. 9 of Benetti et al. [3]), as mentioned above.

These two objects have been extensively monitored spectroscopically also in the infrared. The comparative analysis with the IR spectra of SN 1994D [39] at about day $-8.5,+11.1$ and +29.4 confirms that the overall spectroscopic homogeneity among normal SNe Ia extends to the IR-domain, with small variations. Spectral synthesis on the spectra of SN 2002 bo favors the MgII identification for the $10800 \AA$ line and that of SiII $16910 \AA$ and MgII $16760 / 800 \AA$ (with the SiII dominant) for the broad P-Cygni profile observed 


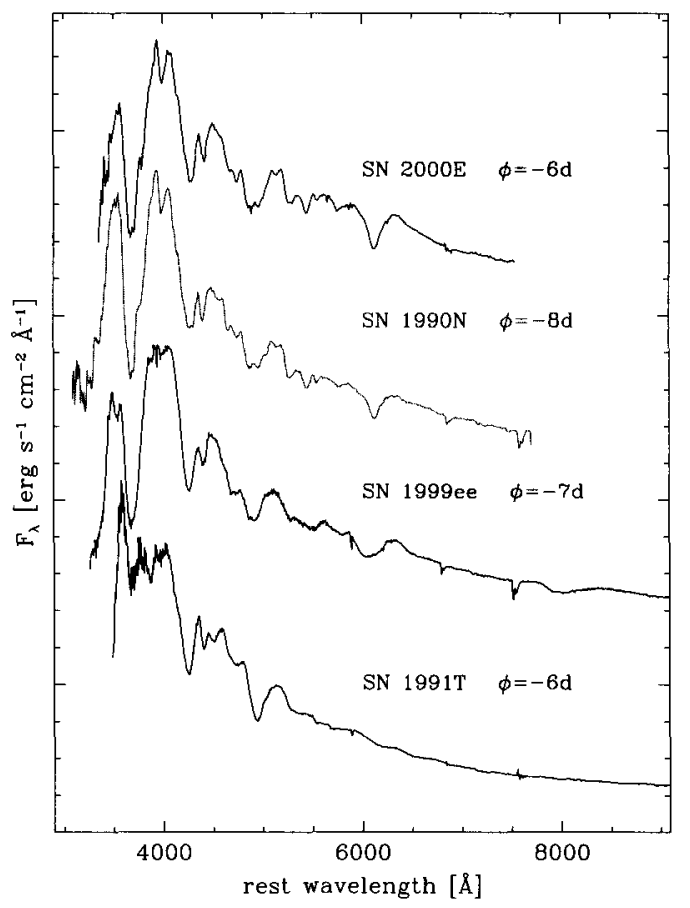

Fig. 1. Comparison of the spectra of SNe 2000E $\left(\Delta m_{15}=0.94 \mathrm{mag}(100 \mathrm{~d})^{-1}\right)$, $1990 \mathrm{~N}\left(\Delta m_{15}=1.05\right), 1999 \mathrm{ee}\left(\Delta m_{15}=0.92\right), 1991 \mathrm{~T}\left(\Delta m_{15}=0.94\right)$, taken about 1 week before maximum light. They have been corrected for extinction and reported to the parent galaxy rest frame.

at $\sim 16000 \AA$. On the contrary, major differences are visible when the comparison is made with peculiar objects, e.g., the faint SN 1999by [23], especially because of the presence of strong $\mathrm{C}$ I and O I lines.

The optical and the IR spectra of SNIa [19] shows different behaviors: 1) while the optical spectrum before maximum is dominated by strong $P$ Cygni profiles of intermediate-mass elements, such as Ca II, Si II, Mg II, S II, the IR is characterized by a smooth, almost featureless continuum; 2) the lines of iron group elements, such as Co II, Fe II and Ni II, emerge in the IR as soon as one week past maximum, definitely earlier than in the visual. This supports the suggestion [51] that the IR photosphere recedes rapidly to the center of the supernova, while at optical wavelengths the greater opacity arising from the higher spectral density of lines keeps the photosphere at higher velocities. At the longer wavelengths the overall emission increases after maximum light as a result of a shift in ionization to lower ionization species, which have greater emissivity in the near IR. It appears, therefore, that the J-band deficit is due to the relative absence of lines in the 12000 $\AA$ region, rather than increasing opacity, and that the secondary maxima 
exhibited by the IR light curves are due to the increasing release of energy through lower optical depth IR transitions. The prominent postmaximum emission features displayed by SNe 1999ee and 2002bo in the $\mathrm{H}$ and $\mathrm{K}$ bands lend support to this scenario [19].

A new powerful tool for understanding the nature of SNIa is spectropolarimetry. SNIa polarization is usually very small, hence difficult to measure. In most cases only upper limits have been provided, in others polarization of the order of $0.2-0.3 \%$ (corresponding to an asphericity of $\sim 10 \%$ ) has been detected before maximum light, likely due to a distorted photosphere or element distribution. Such asphericity may cause a directional dependence of the luminosity and a corresponding dispersion in the brightness-decline relation of SNe Ia. Although this may not jeopardize the use of SNIa as distance indicators, it might intrinsically limit the accuracy reachable via SNIa.

Very interesting spectropolarimetric data have been collected for special SNIa. High polarization $(0.7 \%)$ was found in the subluminous SN 1999by [24], which may suggest a relation between the observed asymmetry and the mechanism that produces this kind of under energetic SNIa. The high velocity components ( $\sim 25000 \mathrm{~km} \mathrm{~s}^{-1}$ ) of the CaII IR triplet observed in SN 2001el have shown a polarization of about $0.7 \%$, much higher than the continuum $(0.2 \%)$ indicating that kinematically and geometrically distinct features can exist in SNIa [58]. High signal-to-noise data for more objects are definitely needed in order to understand if these asymmetries are the rule in SNIa, and to get insights on the geometry of the precursor systems.

A special case is that of SN 2002ic, the first SNIa for which $\mathrm{H}$ has been unequivocally detected [20]. Indeed both the light curve and the spectral line appearance points toward a strong interaction of the SN ejecta with a dense CSM. At the early phase the characteristic spectrum of a SNIa seemed veiled by a strong continuum. With time the spectrum evolved to resemble those of SNe 1997cy and 1999E, which are commonly considered as SNIIn [17, 48, 56]. Also the profile of the $\mathrm{H} \alpha$ emission requires at least two components with different widths to provide a reasonable fit. It has, therefore, been suggested that some SNIIn are the outcome of thermonuclear explosions rather than core collapses. Despite the early claim that these observations were a proof that the progenitor system of SN 2002ic was a binary consisting of a $\mathrm{C} / \mathrm{O}$ WD and an AGB star [20], it may well be that it is the result of the merging of a WD with the core of an AGB star, and that the $\mathrm{H}$ we observe was previously ejected during a common envelope phase [32]. The rarity of such an event and the large amount of $\mathrm{H}$ required favors the latter hypothesis. A polarization of $p \geq 1 \%$ [28] has been detected also in SN 2002 ic.

\section{Core Collapse Supernovae}

Stars of initial mass larger than about $8 \mathrm{M}_{\odot}$ undergo the collapse of the core after burning $\mathrm{H}, \mathrm{He}, \mathrm{C}, \mathrm{Ne}, \mathrm{O}$, Si. From such collapse a SN usually results. 


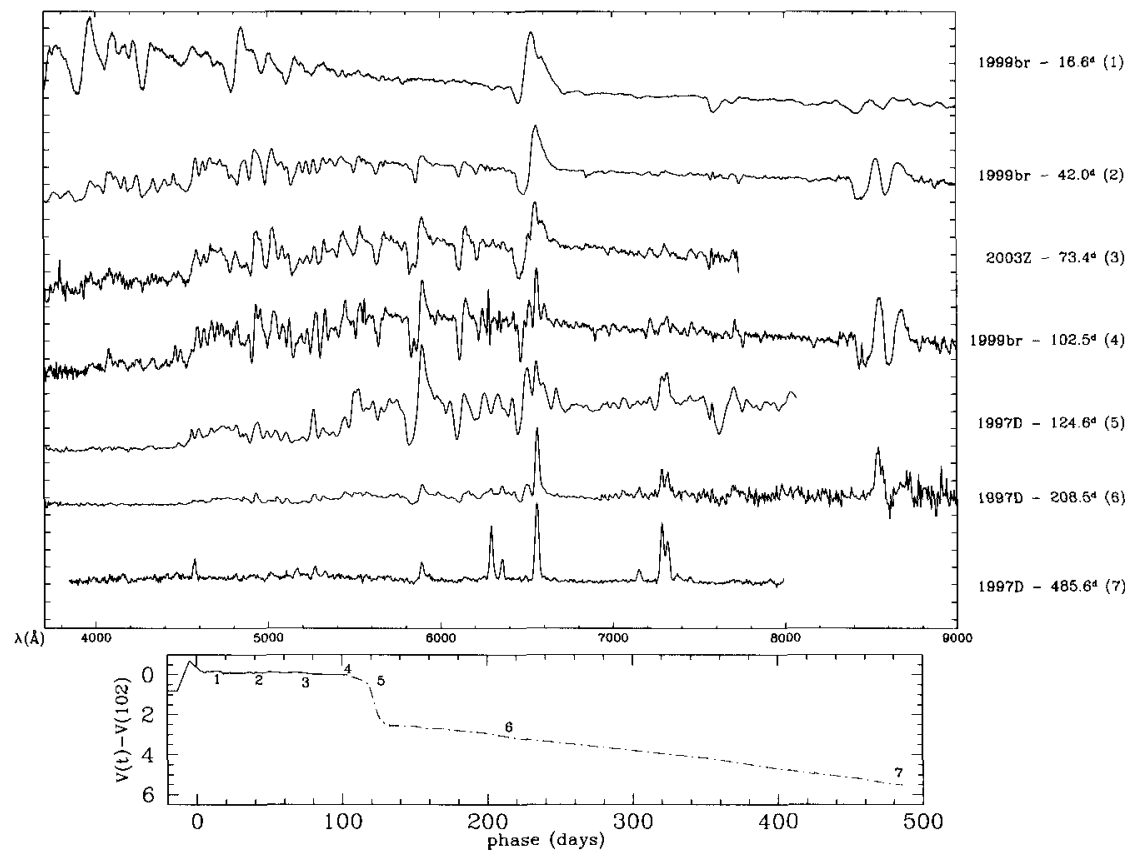

Fig. 2. Spectral evolution of low-luminosity SNII. The objects and the days after the estimated epochs of explosions are marked on the right. In the bottom panel are marked the corresponding epochs on a schematic light curve.

Core collapse SNe can vary considerably in luminosity, spectral lines and time evolution. The main parameter governing the observed diversity is the envelope mass [40]. Stars retaining $\mathrm{H}$ will display the lines of this element during their entire evolution and are called SNII. If the $\mathrm{H}$ mass is large (10-15 $\mathrm{M}_{\odot}$ ) the release of the energy deposited by the shock in the envelope and by the recombination can sustain the luminosity for months, producing the so-called SNII Plateau. If the envelope is less massive $\left(1-2 \mathrm{M}_{\odot}\right)$, stripped by a companion or lost by stellar wind, the SN quickly fades producing the so-called SNII Linear.

In both cases the spectrum evolves from a very blue, featureless continuum short after the burst (color temperature higher than $10^{4} \mathrm{~K}$ ) to one dominated by $\mathrm{H}$ and $\mathrm{He}$ in a few days. Then, as the temperature continues to fall, other low excitation lines of $\mathrm{Na}$ I, Ca II, Fe II, Sc II appear, all with P-Cygni profiles. Subsequently, as the entire star becomes transparent and the light curve settles onto the radioactive tail, the spectrum enters the nebular phase, dominated by $\mathrm{H} \alpha$ and forbidden emission lines of $[\mathrm{O} \mathrm{I}]$, [Ca II], [Fe II] and $\mathrm{Mg} \mathrm{I}]$.

After SN 1987A, the best studied (but somehow atypical) object, whose detailed spectral observations provided evidences of clumps and mixing in the 
ejecta $[21,50]$ as well as of dust formation [9], other SNII have been studied extensively. An interesting case is SN 1999em [1, 11, 18, 29]. The analysis of the $\mathrm{H} \alpha$ structure at photospheric epoch revealed non spherical ejection of ${ }^{56} \mathrm{Ni}$, while the transformation of the [O I] $6300 \lambda$ line profile around day 500 showed that dust formed earlier and at lower velocity than in SN 1987A, probably because of the lower temperature due to a smaller amount of ${ }^{56} \mathrm{Ni}$ ejected. It appears, therefore, that these phenomena are common in core collapse SNe, though with distinctive characteristics for each object.

Core-collapse SNe which have lost most of their $\mathrm{H}$ and even most, or all, their He envelope, are called SNIb and Ic, respectively. Indeed the transition between SNII and SNIb, and between SNIb and SNIc is not sharp. SN1993J, celebrated with this Conference, is the best example (but not the only one) of a SN transforming with age from a type II to a type Ib SN, i.e. from one dominated by $\mathrm{H}$ to one dominated by $\mathrm{He}$, with only residual $\mathrm{H}$ lines. For this reason it is called of type IIb [14].

At a deep scrutiny, $\mathrm{H}$ has been found in the spectra of other SNIb. SN $2000 \mathrm{H}$ is a remarkable case, but Branch et al. [7] have shown that tiny differences in the $H$ mass can vary the optical depths of $H$ lines and make the transition from type Ilb to type Ib SNe. Similarly, an increasing number of SNIc show evidence of He, e.g., SN 1990B, SN 1987M and even the prototypical SN1994I [8, 12]. Again, it appears that moderate difference in the He mass can explain the spectroscopic difference between typical SNIb and SNIc [7]. Different is the case of SN 1999cq, in which He with expansion velocity much slower than other lines, points to the interaction of the ejecta with a dense shell of almost pure He originating from stellar wind or mass transfer to a companion [33].

\subsection{News on Core Collapse SN Spectra}

In the last years new light on the lower end of the luminosity function of SNII has been shed $[42,60]$. SN1997D showed from discovery unprecedented properties. The expansion velocity deduced from the displacement of the absorption was extremely low and the continuum intrinsically red. Moreover, the luminosity was very low both at discovery and in the nebular stage, indicating the ejection of a very little amount of ${ }^{56} \mathrm{Ni}$ [55].

Four new objects with similar properties have been recently added [42]. Although the observations of individual objects are erratic and incomplete, because hampered by the faintness of the SNe, taken together these data show a common evolutionary path. Fig. 2 summarizes the spectroscopic evolution: during the first 50 days (spectra 1 and 2) the spectra change from a continuum dominated by Balmer lines to a more complex appearance with strong $\mathrm{Na}$ I, $\mathrm{Ba}$ II and Ca II. The expansion velocities, lower than in normal SNII, decrease from 5000 to $3000 \mathrm{~km} \mathrm{~s}^{-1}$. During the second half of the plateau (spectra 3 and 4 ) these peculiarities strengthen significantly: the absorption troughs move to even lower velocities, down to $1000-1500 \mathrm{~km} \mathrm{~s}^{-1}$, the continuum 
becomes redder and low excitation lines of $\mathrm{Ba} \mathrm{II,} \mathrm{Sc} \mathrm{II,} \mathrm{Fe} \mathrm{II,} \mathrm{Sr} \mathrm{II} \mathrm{and}$ Ti II appear. In particular, the Ba II lines become the strongest features of the entire spectrum, even stronger than $\mathrm{H} \alpha$. During the post plateau decline (spectrum 5) the transition to the nebular phase begins and forbidden lines (e.g., [Ca II] 7291-7323 $\AA$ ) emerge. The spectra of the latest epochs (6 and 7) resemble those of normal SNII, with the usual (though narrower) emissions of $\mathrm{H} \alpha, \mathrm{Na}$ I, [O I], [Ca II], [Fe II], Mg I].

Pastorello et al. [42] conclude that low-luminosity $\mathrm{CC}-\mathrm{SNe}$ are similar to typical $\mathrm{CC}-\mathrm{SNe}$ in having a clear plateau, lasting for $\sim 100$ days, followed by a late-time decline driven by the decay of ${ }^{56} \mathrm{Co}$, and typical spectral lines at all phases. However, they keep distinctive characteristics in that: 1) during the plateau phase the luminosity is at least a factor 10 times less than in typical CC-SNe; 2) the expansion velocity is unusually low at all epochs; and 3 ) the mass of ${ }^{56} \mathrm{Co}$ which drives the late-time tail is at least a factor $\sim 10$ lower than normal.

Important advances have been obtained also at the opposite extreme, i.e. for luminous and energetic SNe, often called hypernovae. In particular, large interest have received a number of SNe associated to GRBs. The first and best studied case is SN 1998bw which coincided in time and space with GRB980425 [15] and was a powerful radio and X-ray emitter. In the optical most of its peculiarity stayed in the unprecedented broadness of the spectral features corresponding to expansion velocities of the ejecta as high as $3 \times 10^{4}$ $\mathrm{km} \mathrm{s}^{-1}$ at maximum light [43]. The high kinetic energy together with the high luminosity indicated an explosion energy of about $5 \times 10^{52} \mathrm{erg}$, if spherical symmetry is assumed [25]. Detailed spectral modeling $[6,25,27,36]$ has shown that the apparent emissions, which at maximum peaked around 5000 , 6300 and $8500 \AA$, were actually low opacity regions of the spectra from which photons could escape. Lines of Si II, O I, Ca II and FeII have been identified, as well as He I $10830 \AA$.

Only during the nebular phase SN 1998bw reentered into the conventional taxonomical scheme with [O I], Mg I], [Fe II], [Ca II] emissions which confirmed the early hypothesis that it was a peculiar case of SNIc. Also in the nebular phase the lines were unusually broad $\left(9800 \pm 500 \mathrm{~km} \mathrm{~s}^{-1}\right.$ on day 201 [43]).

After SN 1998bw several other hypernovae have been recognized, some were SNIc bearing strong resemblance to SN 1998bw but with smaller KE (1997ef, 1997dq, 1999as, 2002ap [26, 27, 34, 37]), other were SNIIn (1997cy and 1999E $[17,48,56]$ ), which displayed narrow $\mathrm{H} \alpha$ on the top of broad wings and broad light curves, clear indications of ejecta-CSM interaction. For some of them the possible association with GRB has been claimed but no firm conclusion was reached. Other cases of possible SN-GRB association have been reported on the basis of 'bumps' detected in the light curves of GRB afterglows. The early spectroscopy of these bumps was intriguing but not conclusive $[10,16]$. 
Excitement mounted when, short before this Conference, spectra of the rebrightening of the afterglow of the nearby GRB030329 $(\mathrm{z}=0.1685)$ were secured $[35,52]$. Already one week after the burst a SN spectrum, with emissions at approximately 5000 and $4200 \AA$, was detected and it dominated over the power-law continuum few days later (day $\sim 11$ ). This $\mathrm{SN}$, named 2003dh, resembled, both as spectral evolution and light curve, SN 1998bw at the corresponding epoch from the associated GRB. A more accurate analysis and spectral modeling suggested that SN 2003dh was intermediate between SN 1998bw and SN $1997 \mathrm{ef}$, as to kinetic energy and ${ }^{56} \mathrm{Ni}$ production [38] and pointed out the need for asymmetric explosions. Whatever the physics of the explosion, this new example provides solid evidence that at least some GRBs arise from core-collapse SNe and opens new frontiers in the SN research.

Acknowledgement. This research is supported in part by the European Community's Human Potential Programme under contract HPRN-CT-2002-00303, and grant Cofin 2001021149 of the Italian Ministry of Education, University and Research.

\section{References}

1. E. Baron et al. : Astrophys. J. 545, 444 (2000)

2. S. Benetti et al. : Mon. Not. R. Astron. Soc. 322, 361 (2001)

3. S. Benetti et al. : astro-ph 0309665 (2003)

4. D. Branch, S. Van Den Bergh: Astron. J. 105, 2231 (1993)

5. D. Branch et al. : Pub. Astron. Soc. Pacific 107, 1019 (1995)

6. D. Branch: In: Supernovae and Gamma-Ray Bursts eds. M. Livio, N. Panagia (Cambridge Univ, Press: Cambridge, 2001) p. 96

7. D. Branch et al. : Astrophys. J. 566, 1005 (2002)

8. A. Clocchiatti et al. : Astrophys. J. 462, 462 (1996)

9. I.J. Danziger et al. : In: Supernova 1987 A and Other Supernovae, ed. I.J. Danziger, K. Kjar (ESO Conf. and Workshop Proc.) p. 217

10. M. Della Valle et al. : Astron. Astrophys. 406, 33 (2003)

11. A. Elmhamdi et al. : Mon. Not. R. Astron. Soc. 338, 939 (2003)

12. A.V. Filippenko et al. : Astrophys. J. Lett. 450, L11 (1995)

13. A.V. Filippenko: Ann. Rev. Astron. Astrophys. 35, 309 (1997)

14. A.V. Filippenko, T. Matheson: "Optical, Ultraviolet, and Infrared Observations of SN 1993J." In: this Proceedings

15. T.J. Galama et al. : Nature 395, 670 (1998)

16. P.M. Garnavich et al. : Astrophys. J. 582, 924 (2003)

17. L. Germany et al. : Astrophys. J. 533, 320 (2000)

18. M. Hamuy et al. : Astrophys. J. 558, 615 (2001)

19. M. Hamuy et al. : Astron. J. 124, 417 (2002)

20. M. Hamuy et al. : Nature 424, 651 (2003)

21. R.W. Hanuschik et al. : Mon. Not. R. Astron. Soc. 243, 41 (1988)

22. K. Hatano et al. : Astrophys. J. Lett. 543, L49 (2000)

23. P. Höflich et al. : Astrophys. J. 568, 791 (2002) 
24. D.A. Howell et al. : Astrophys. J. 556, 302 (2001)

25. K. Iwamoto et al. : Nature 395, 672 (1998)

26. K. Iwamoto et al. : Astrophys. J. 534, 660 (2000)

27. K. Iwamoto et al. : "SN 1998bw and Hypernovae." In: Supernovae and GammaRay Bursters, ed. K.W. Weiler (Springer: Berlin, 2003) pp. 243-281

28. K.S. Kawabata et al. : IAUC 8161 (2003)

29. D.C. Leonard et al. : Pub. Astron. Soc. Pacific 114, 35 (2002)

30. W. Li, A.V. Filippenko et al. : Pub. Astron. Soc. Pacific 113, 1178 (2001)

31. W. Li et al. : astro-ph 0301428 (2003)

32. M. Livio, A.G. Riess: Astrophys. J. Lett. 594, L93 (2003)

33. T. Matheson et al. : Astron. J. 119, 2303 (2000)

34. T. Matheson et al. : Astron. J. 121, 1648 (2001)

35. T. Matheson et al. : astro-ph 0307435 (2003)

36. P. Mazzali et al. : Astrophys. J. 559, 1047 (2001)

37. P. Mazzali et al. : Astrophys. J. Lett. 572, L61 (2002)

38. P. Mazzali et al. : astro-ph 0309555 (2003)

39. W.P.S. Meikle et al. : Mon. Not. R. Astron. Soc. 281, 263 (1996)

40. K. Nomoto, K. Iwamoto, T. Suzuki: Phys. Rep. 256, 173 (1995)

41. P. Nugent et al. : Astrophys. J. Lett. 455, L147 (1995)

42. A. Pastorello et al. : astro-ph 0309264 (2003)

43. F. Patat et al. : Astrophys. J. 555, 917 (2001)

44. S. Perlmutter et al: Astrophys. J. 483, 565 (1997)

45. M.M. Phillips: Astrophys. J. Lett. 413, L105 (1993)

46. M.M. Phillips: Astron. J. 118, 1766 (1999)

47. A,G. Riess et al. : Astron. J. 116, 1009 (1998)

48. L. Rigon et al. : Mon. Not. R. Astron. Soc. 340, 19 (2003)

49. B.P. Schmidt et al. : Astron. J. 107, 1444 (1994)

50. J. Spyromilio et al. : Mon. Not. R. Astron. Soc. 263, 530 (1993)

51. J. Spyromilio et al. : Mon. Not. R. Astron. Soc. 266, L17 (1994)

52. K.Z. Stanek et al. : Astrophys. J. 591, 17 (2003)

53. R.C. Thomas et al. : astro-ph 0302260 (2003)

54. M. Turatto, et al: Mon. Not. R. Astron. Soc. 283, 1 (1996)

55. M. Turatto, et al: Astrophys. J. Lett. 498, L129 (1998)

56. M. Turatto, et al: Astrophys. J. Lett. 534, L57 (2000)

57. M. Turatto: "Classification of Supernovae." In: Supernovae and Gamma-Ray Bursters, ed. K.W. Weiler (Springer: Berlin, 2003) pp. 21-36

58. L. Wang et al. : Astrophys. J. 591, 1110 (2003)

59. J.C. Wheeler, S. Benetti: In: Allen's Astrophysical Quantities, ed. A.N. Cox (Springer: New York, 2000) p. 451

60. L. Zampieri et al. : Mon. Not. R. Astron. Soc. 338, 711 (2003) 